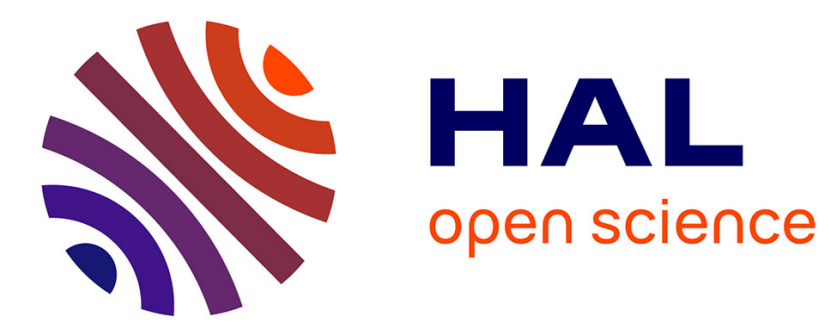

\title{
Topological Analysis of High Velocity Turbulent Flow
}

Thibault Bridel-Bertomeu, Benjamin Fovet, Julien Tierny, Fabien Vivodtzev

\section{To cite this version:}

Thibault Bridel-Bertomeu, Benjamin Fovet, Julien Tierny, Fabien Vivodtzev. Topological Analysis of High Velocity Turbulent Flow. IEEE Symposium on Large Data Analysis and Visualization, Oct 2019, Vancouver, Canada. hal-02321841

\section{HAL Id: hal-02321841 \\ https://hal.science/hal-02321841}

Submitted on 21 Oct 2019

HAL is a multi-disciplinary open access archive for the deposit and dissemination of scientific research documents, whether they are published or not. The documents may come from teaching and research institutions in France or abroad, or from public or private research centers.
L'archive ouverte pluridisciplinaire HAL, est destinée au dépôt et à la diffusion de documents scientifiques de niveau recherche, publiés ou non, émanant des établissements d'enseignement et de recherche français ou étrangers, des laboratoires publics ou privés. 


\section{Topological Analysis of High Velocity Turbulent Flow}

\author{
Thibault Bridel-Bertomeu* \\ CEA, France \\ Benjamin Fovet \\ CEA, France
}

\author{
Julien Tierny \\ CNRS, Sorbonne Université, LIP6, France
}

Fabien Vivodtzev
CEA, France
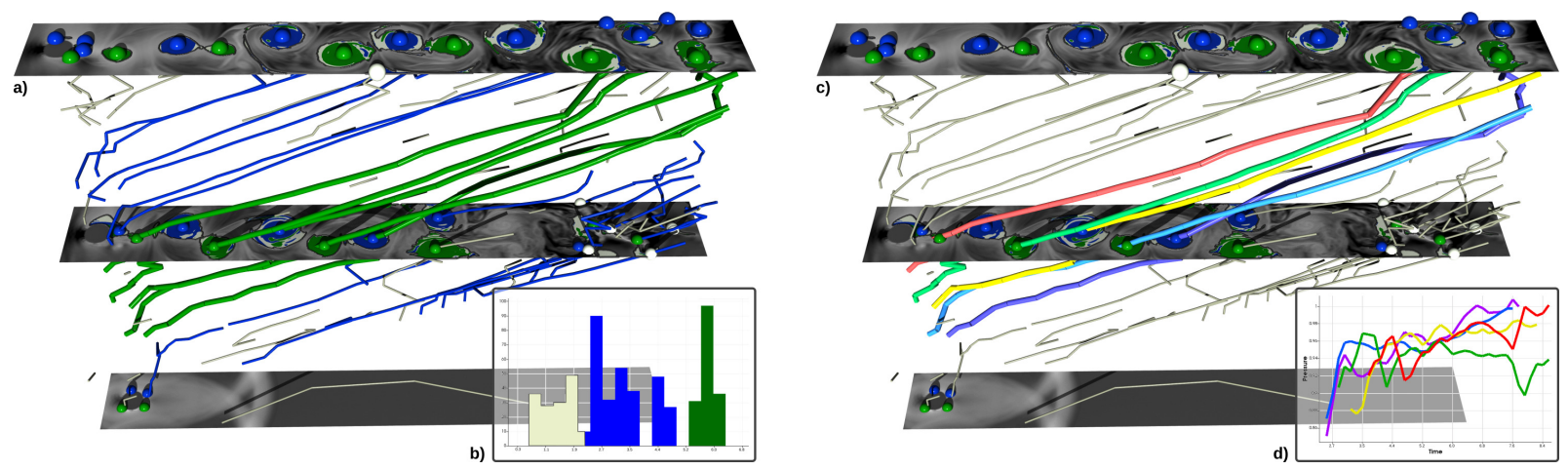

Figure 1: Topology-based tracking and analysis of vortices (flow density, height is time). The vortex trajectories (a) exhibit 3 modes in terms of duration, corresponding to the 3 colors in the inset histogram (b): noise (white), intermediate length (blue) and full length (green). The 4 longest trajectories (c) are used to analyse the time evolution of flow density at the center of vortices, indicating an increase tendency. See the second companion video (blue spheres: counter-clockwise vortices, green spheres: clockwise vortices).

\section{Abstract}

In order to guarantee the performances of complex systems, the CEA is driving large numerical simulations in various fields such as thermomechanics, electromagnetism and aerodynamics. Due to the size of the problems and the use of High Performance Computing approaches, large and complex datasets need to be explored to understand the physical phenomena. This paper focuses on the exploration of a compressible turbulent 2D flow, to better understand the flight behavior of an object. Topological data analysis (TDA) is used to improve understanding and avoid costly traditional methods such as 3D modal decomposition algorithms or highly technical hydrodynamic stability codes. The attention is put on the large eddies shed behind a cylinder hit by a crossflow. Thanks to TDA the tracking of the eddies, the identification of their origin and the evolution of their amplitude with the downstream distance are facilitated.

\section{BACKGROUND}

This work addresses the analysis of large structures in computational fluid dynamics (CFD, Sect. 1.1) with topological data analysis (TDA, Sect. 1.2). It constitutes a first step towards the application of TDA for the analysis of more complex flows (flight of a projectile).

\subsection{Numerical simulation}

The data generated for the present study (detailed in Sect. 2) comes from a direct solver of the Navier-Stokes (NS) equations. These equations can be written in tensor form as [16]:

$$
\left\{\begin{aligned}
\rho_{t}+\nabla \cdot(\rho \mathbf{V}) & =0 \\
(\rho \mathbf{V})_{t}+\nabla \cdot(\rho \mathbf{V V}) & =-\nabla p+\nabla \cdot \mathbf{S} \\
(\rho E)_{t}+\nabla \cdot(\rho E \mathbf{V}) & =\nabla \cdot((\mathbf{S}-p \mathbf{I}) \cdot \mathbf{V})+\nabla \cdot \mathbf{q}
\end{aligned}\right.
$$

*e-mail: thibault.bridel-bertomeu@cea.fr

†e-mail: benjamin.fovet@cea.fr

¥e-mail: julien.tierny@ sorbonne-universite.fr

§e-mail: fabien.vivodtzev@cea.fr

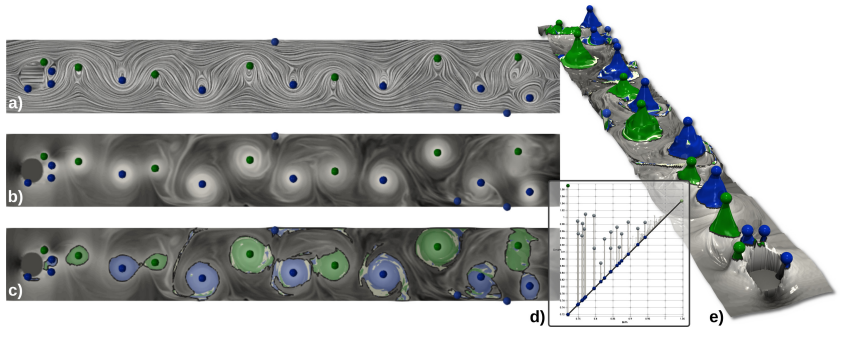

Figure 2: Topological analysis of a single time step. a) Line Integral Convolution [3] of the input vector field. b) Flow density. c) Merge tree based vortex segmentation (spheres: center). d) Persistence diagram. e) Terrain view. See the first companion video (blue: counterclockwise rotations, green: clockwise rotations).

where $\rho$ is the density of the fluid, $\mathbf{V}$ its velocity vector, $p$ its pressure, $E$ its energy and $\mathbf{S}$ and $\mathbf{q}$ represent respectively the effects of viscous strain and heat transfer onto the motion and the energy of the fluid. During the post-processing of the data (described in section 2) we will be most interested in $\rho$ and $\mathbf{V}=[u, v, w]^{t}$. These equations are solved using a conservative finite volume algorithm on a Cartesian grid (see e.g. [15]), and any complex boundary located inside the domain is handled using a ghost-cell based immersed boundary method $[5,10]$. The Roe approximate solver [15] has been used for the numerical fluxes, with the left and right states being interpolated using a $5^{\text {th }}$ order WENO scheme [9] while timestepping has been kept explicit and the time derivative discretized using a $3^{\text {rd }}$ order, strong stability-preserving Runge-Kutta algorithm [7].

\subsection{Topological Data Analysis}

Topological Data Analysis is a recent set of techniques [6,12], which focus on structural features in data. For our analysis, we used several established techniques, readily available in the "Topology ToolKit" (TTK) [13]. To adjust the parameters of our analysis, we first considered a single time step (Fig. 2). In particular, vortices were identified as local minima of $\rho$ and their region of influence given by a merge tree based segmentation [8]. The rotation direction of each vortex is estimated by the sign of the orthogonal component 

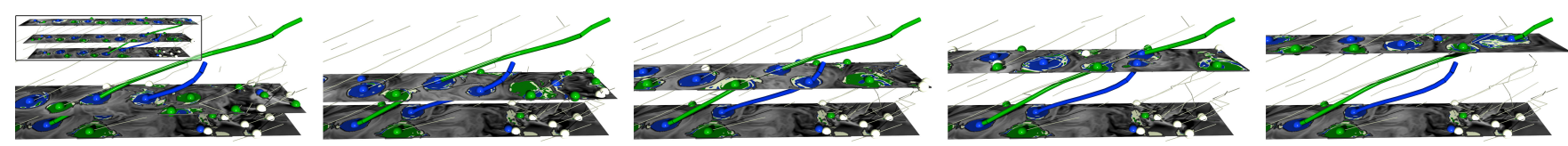

Figure 3: The two trajectories which maximize lateral movement (blue and green) correspond to 2 clockwise vortices which merge together. Due to the irregularity of this von Kármán street, vortices interact with each other. When they rotate in the same direction, interacting vortices attract each other in a spiral movement (hence the high lateral movement) and eventually merge, as captured by our analysis. See the third companion video.
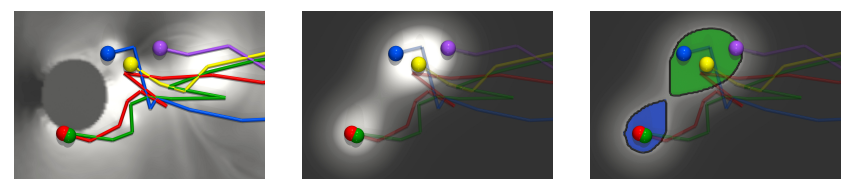

Figure 4: Zoom in the shear-layer region behind the obstacle (left) Clockwise vortices (green) seem to be generated in the low-pressure region whereas anti-clockwise (blue) eddies seem to be born in the high pressure region (merge tree segmentation, right, of a Gaussian density estimation of the long trajectory starts, middle).

of the curl of $\mathbf{V}$. To discard noise, vortex importance within a single time step was assessed by the topological persistence [6] of the corresponding minimum of $\rho$. To estimate an appropriate persistence threshold, we considered the persistence diagram (Fig. 2(d)) which represents each minimum, denoting a vortex, as a vertical bar, whose height denotes the density amplitude of the vortex. Bars near the diagonal, corresponding to noisy structures, can easily be isolated (persistence below $10 \%$ of the global value range) and the data can be simplified [14] to account for this noise removal.

Next, minima of $\rho$ are tracked through time by estimating an optimal assignment based on the Wasserstein metric between consecutive time steps [11] (Fig. 1, geometrical lifting coefficient: 0.005).

\section{Case Study}

This section presents our turbulent flow data and its interpretation.

\subsection{Data description}

The considered use case is a von Kármán street (Fig. 2): fluid enters the domain behind a cylinder obstacle (left) at Mach 0.475 and with a Reynolds number of $10^{5}$, and can only exit at the other end of the domain, the rest of the boundaries being set as periodic. The computation is run up to $t_{f}=8.5 \times 10^{-3}$ seconds in physical time by 15,625 steps. A total of 625 snapshots have been taken in order to realize the present analysis, separated from each other by a constant timestep of $1.35 \times 10^{-5}$ seconds, i.e. at a frequency of about $75 \mathrm{kHz}$. Each snapshot stores $\rho, \rho \mathbf{V}$ and $\rho E$ (Sect. 1.1) in double precision on a $5000 \times 4002 \mathrm{D}$ grid, yielding a total amount of data of $14 \mathrm{~GB}$.

\subsection{Interpretation}

The extraction and temporal tracking of the vortices thanks to TDA took 215 seconds overall (Xeon CPU, $2.6 \mathrm{GHz}, 2 \times 6$ cores). The vortex tracking provides a valuable feature representation for further analysis and interpretation. In particular, the 4 longest trajectories (in color, Fig. 1) are used in the remainder to estimate further statistics. For instance, we use the startup time of these trajectories to estimate the frequency of the vortex shedding $(1,500 \mathrm{~Hz})$, which matches the theoretical expectations [1] and validates the relevance of our analysis pipeline.

The time evolution of the $Y$ coordinate of a vortex center is used to denote its lateral movement, which assesses how much a vortex trajectory deviates from a straight line. The 2 trajectories which maximize their lateral movement are shown in Fig. 3. These correspond to two interacting vortices rotating in the same direction, entering a coupled spiral movement and eventually merging together. This indicates that this kind of vortex merging can be systematically tracked thanks to TDA. Our analysis also enables to investigate the vortex start points, also known as wavemakers (Fig. 4). In hydrodynamics, determining wavemaker locations often requires stability analyses $[2,4]$ that are tricky to develop and manipulate. This information can be, however, of great importance, for instance for the identification and resolution of sources of vibrations in an engine. Here, by considering a merge tree segmentation of a density estimation of the trajectory start points, locations for wavemakers can be estimated, which could open new horizons in future work.

\section{CONCLUSION AND FUtURE WORK}

In this work, we showed that established methods from TDA could be used to provide an analysis support for the detailed investigation of high velocity compressible turbulent flow, which would be difficult to conduct with traditional methods. In particular, our framework enabled to estimate vortex shedding frequency, wavemaker locations as well as to extract vortex merging events. In future work, we plan to transpose this analysis to the flight of a projectile in an in-situ setting, with more challenging data sizes and resolutions.

\section{ACKNOWLEDGMENTS}

This work is partially supported by the European Commission grant H2020FETHPC-2017 "VESTEC" (ref. 800904).

\section{REFERENCES}

[1] R. D. Blevins. Flow-induced vibration. New York, Van Nostrand Reinhold Co., 1977. 377 p., 1977.

[2] T. Bridel Bertomeu. Investigation of unsteady phenomena in rotor/stator cavities using Large Eddy Simulation. PhD thesis, 2016.

[3] B. Cabral and L. C. Leedom. Imaging vector fields using line integral convolution. Technical report, LLNL, 1993.

[4] S. Chandrasekhar. Hydrodynamic and hydromagnetic stability. Courier Corporation, 2013.

[5] C. Chi, B. J. Lee, and H. G. Im. An improved ghost-cell immersed boundary method for compressible flow simulations. International Journal for Numerical Methods in Fluids, 83(2):132-148, 2017.

[6] H. Edelsbrunner and J. Harer. Computational Topology: An Introduction. AMS, 2009.

[7] S. Gottlieb, D. I. Ketcheson, and C.-W. Shu. High order strong stability preserving time discretizations. J. of Sci. Comp., 2009.

[8] C. Gueunet, P. Fortin, J. Jomier, and J. Tierny. Task-based Augmented Contour Trees with Fibonacci heaps. IEEE TPDS, 2019.

[9] G.-S. Jiang and C.-W. Shu. Efficient implementation of weighted eno schemes. Journal of computational physics, 126(1):202-228, 1996.

[10] R. Mittal and G. Iaccarino. Immersed boundary methods. Annu. Rev. Fluid Mech., 37:239-261, 2005.

[11] M. Soler, M. Plainchault, B. Conche, and J. Tierny. Lifted wasserstein matcher for fast and robust topology tracking. In IEEE LDAV, 2018.

[12] J. Tierny. Topological Data Analysis for Scientific Visualization. Springer, 2018.

[13] J. Tierny, G. Favelier, J. A. Levine, C. Gueunet, and M. Michaux. The Topology ToolKit. IEEE TVCG, 2017. https:// topology-tool-kit.github.io/.

[14] J. Tierny and V. Pascucci. Generalized topological simplification of scalar fields on surfaces. IEEE TVCG, 2012.

[15] E. F. Toro. Riemann solvers and numerical methods for fluid dynamics: a practical introduction. Springer Science \& Business Media, 2013.

[16] F. M. White and I. Corfield. Viscous fluid flow, vol. 3. McGraw-Hill New York, 2006. 